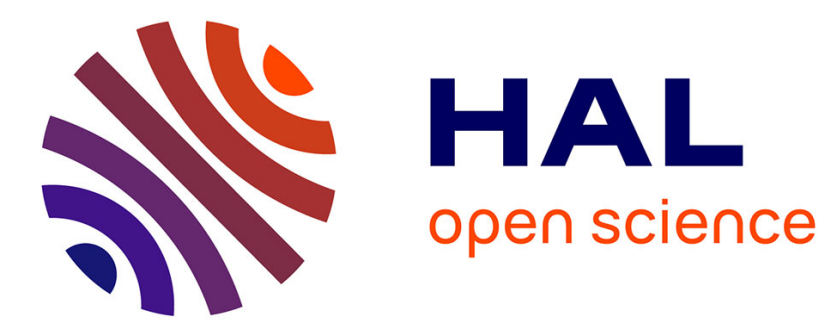

\title{
À la recherche des Orientaux dans l'œuvre d'Athénée
}

Dominique Lenfant

\section{To cite this version:}

Dominique Lenfant. À la recherche des Orientaux dans l'œuvre d'Athénée. Archimède: archéologie et histoire ancienne, 2017, 4, pp.60-67. halshs-01589056

\section{HAL Id: halshs-01589056 \\ https://shs.hal.science/halshs-01589056}

Submitted on 18 Sep 2017

HAL is a multi-disciplinary open access archive for the deposit and dissemination of scientific research documents, whether they are published or not. The documents may come from teaching and research institutions in France or abroad, or from public or private research centers.
L'archive ouverte pluridisciplinaire HAL, est destinée au dépôt et à la diffusion de documents scientifiques de niveau recherche, publiés ou non, émanant des établissements d'enseignement et de recherche français ou étrangers, des laboratoires publics ou privés. 


\section{ARCHIMĖDE No4}

\section{DOSSIER THÉMATIQUE 1 : NOMMER LES « ORIENTAUX » DANS L'ANTIQUITÉ}

1 Dominique LENFANT, Agnès MOLINIER ARBO et Pascale GIOVANNELLI-JOUANNA Nommer les « Orientaux » dans l'Antiquité : présentation du dossier

6 Luca MACALE et Francesco MARI

Le lexique grec de I'Oriental dans la poésie lyrique archaïque et chez Eschyle

19 Dominique LENFANT

Les « Asiatiques » du traité hippocratique Airs, Eaux, Lieux ont-ils été les premiers « Orientaux »?

26 Yannick MULLER

Le monde « oriental » et ses habitants chez Thucydide

35 Emanuele PULVIRENTI

Des désignations des «Orientaux » chez Xénophon ? Le cas des Helléniques et de I'Anabase

45 Pascale GIOVANNELLI-JOUANNA

Isocrate et I'ennemi commun des Grecs : désignation et représentation des peuples d'Asie dans le corpus isocratique

54 Charlotte LEROUGE-COHEN

Aristote, la Politique et les « habitants de l'Asie »

60 Dominique LENFANT A la recherche des Orientaux dans l'œuvre d'Athénée

68 Jean-Luc VIX L'Orient chez Ælius Aristide

73 Agnès MOLINIER ARBO Ammien Marcellin. L'Orient et les Orientaux dans l'Empire au Iv siècle

80 Agnès MOLINIER ARBO Le vocabulaire de I'Orient et de I'Oriental dans I'Histoire Auguste. Regards d'un Romain sur l'Est de l'Empire à la fin du Iv ${ }^{\mathrm{e}}$ siècle

87 DOSSIER THÉMATIQUE 2 : PRYTANÉE ET REGIA

155 ACTUALITÉ DE LA RECHERCHE : DYNAMIQUES HUMAINES ANCIENNES

216 VARIA

\section{LA CHRONIQUE D’ARCHIMÈDE}

\section{@creative @(1) (2)}




\title{
À LA RECHERCHE DES ORIENTAUX DANS L'CEUVRE D'ATHÉNÉE
}

\author{
Dominique LENFANT \\ Professeure d'histoire grecque \\ Université de Strasbourg \\ UMR 7044 Archimède \\ dlenfant@unistra.fr
}

\section{RÉSUMÉ}

Bien que les Deipnosophistes véhiculent des images stéréotypées de rois d'Asie, on n'y trouve pas de désignation générale des Orientaux en tant qu'ensemble uni et continu qui s'opposerait au groupe dont relèvent les locuteurs. L'analyse requiert de distinguer le vocabulaire des citations de celui d'Athénée lui-même. Ce dernier ne suppose aucune dichotomie entre Occidentaux et Orientaux, ne donne aucune valeur structurante à l'appellation d'Asiatiques employée par certaines de ses sources, ni même à celle de barbares : il se distingue de plusieurs de ses contemporains par ses emplois de bar-

MotS-CLÉS

Athénée de Naucratis, Asie,

Asiatiques,

Orient,

orientalisme,

Grecs,

barbares,

vocabulaire grec. baros, peu nombreux, plus neutres que péjoratifs, en grande partie vidés de contenu politique, voire moral, et nullement voués à conforter l'idée d'une dichotomie générale de I’humanité.
Although Athenaeus' Deipnosophists certainly conveys stereotyped images of Asian kings, it does not provide any overarching term for Eastern people that designates them as a unified whole opposite to the speakers' own group. Analysis of this issue must distinguish the vocabulary of the quotations from that of Athenaeus himself. The latter does not imply any dichotomy between Western and Eastern people. He gives no structuring value to the appellation "Asians," which is used in some of his sources, nor to that of "barbarians" either: compared to several of his contemporaries, he uses the word barbaros infrequently, and usually with a neutral meaning that has largely lost its political and even its moral content, and is not at all used to back up the idea of a general dichotomy between human beings.
Keywords Athenaeus of Naucratis, Asia, Asians, Orient, orientalism, Greeks,

Barbarians, Greek vocabulary. 
Dans ses Deipnosophistes, œuvre inclassable écrite à Rome à la fin $d u \mathrm{II}^{\mathrm{e}}$ siècle de notre ère, Athénée de Naucratis fait, en quinze livres, le récit d'un long banquet tout à fait singulier : il en décrit le service et les plats, mais il rapporte surtout les échanges verbaux entre les convives, qui citent ou évoquent des centaines de textes de la littérature grecque, d'Homère à l'époque hellénistique, évoquant de près ou de loin les pratiques du banquet. Ces usages relèvent de sociétés diverses, tant grecques que non grecques, dont certaines que nous qualifierions d'orientales [1].

L'un des livres les plus connus de cet ouvrage est le douzième, celui qu'il consacre au thème de la truphê, goût de I'abandon aux plaisirs et source de mollesse que les Grecs jugeaient volontiers typiques des rois d'Asie [2]. Ce livre nous a même transmis des portraits hauts en couleur de rois orientaux féminisés, au premier rang desquels celui de I'Assyrien Sardanapale, souverain qui, tout en jouissant d'immenses trésors d'or et d'argent, ne quittait pas l'intérieur de son palais, fuyant les viriles activités de la guerre et de la chasse tout comme le regard de ses sujets, préférant vivre à la manière d'une femme, paré et maquillé comme ses concubines et s'adonnant comme elles au travail de la laine [3]. En dehors même de ce personnage, qui s'inscrit dans une longue lignée dont il représente l'apogée, l'auteur affirme que le premier peuple à s'être rendu célèbre par sa truphê est celui des Perses [4]. Les Deipnosophistes paraissent donc avoir fortement contribué à diffuser une image stéréotypée des Orientaux.

Pour la recherche qui fait l'objet du présent dossier, à savoir l'émergence ou non d'une désignation générale des Orientaux en tant que groupe, I'ouvrage présente ainsi deux particularités, dont I'une fait l'intérêt, I'autre la difficulté. La première est dans son objet, qui embrasse une très grande étendue géoculturelle et propose au lecteur un panorama si large que, s'il supposait une dichotomie entre Occidentaux et Orientaux, cette dernière devrait être sensible. La dimension diachronique augmente encore l'intérêt, puisque les «Orientaux » de référence ne sont pas toujours les mêmes, allant des Assyriens aux Parthes
[1] Les Deipnosophistes ont été édités par KAIBEL 18871890 et plus récemment par OLSON 2006-2012 (avec apparat critique sélectif et traduction anglaise). Pour une présentation de l'ouvrage, de la place qu'y occupent les Perses et de la bibliographie essentielle, LENFANT 2011 (on peut ajouter maintenant J tions générales).

[2] Sur la truphê et sa place dans les Deipnosophistes
d'Athénée, cf. Gambato 2000 et Lenfant 2007a.

[3] Athénée, XII, 528f-529d.

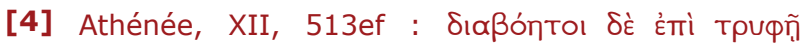

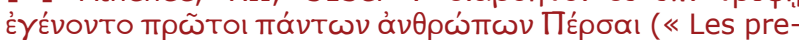
miers au monde à s'être rendus célèbres pour leur truphê ont été les Perses »). Il semble qu'Athénée n'ait pas une vision très claire de I'antériorité des Assyriens comme Sardanapale sur les Perses. 
contemporains de l'auteur [5]. Ce corpus paraît donc à première vue un champ privilégié pour se mettre en quête d'un éventuel terme suggérant en la matière continuité et unité (« rois d'Asie », « peuples d'Asie », « Asiatiques » ou «Orientaux »). La source de difficulté vient, quant à elle, de la nature et de la construction de l'ouvrage : Athénée prétend relater un banquet récent auquel il a pris part en compagnie d'amis lettrés, mais les citations peuvent, quant à elles, remonter à Homère - près d'un millénaire plus tôt - ou être plus récentes. Dans la plupart des cas, elles sont d'époque classique et hellénistique, donc déjà antérieures de plusieurs siècles. Or, durant tous ces siècles, les rapports avec l'Est méditerranéen n'ont pas manqué d'évoluer. De plus, en dehors de la narration du banquet et des citations que I'on peut situer dans le temps, il est une catégorie textuelle intermédiaire. Ce sont les paraphrases de textes anciens [6] et les formules par lesquelles Athénée introduit ses citations. L'auteur peut alors reformuler ce qui était dans sa source en en changeant les mots : on en trouve divers exemples avérés quand on peut comparer la paraphrase avec la source citée, par exemple Hérodote ou Xénophon. Il arrive ainsi qu'Athénée évoque la truphê d'un personnage en se référant à Hérodote, qui, quant à lui, n'employait pas ce terme péjoratif [7]. C'est dire si la prudence est requise quand il s'agit d'interpréter le vocabulaire employé dans les Deipnosophistes.

On peut dire que deux grandes époques sont à distinguer dans le texte, tant du point de vue de I'énonciation que de la période de référence : il y a le passé des citations et le présent des deipnosophistes. Les citations proprement dites témoignent d'usages linguistiques antérieurs. Ainsi, quand I'historien Charès évoque «les Perses, les Mèdes et tous les Asiatiques ('Aoıavoí) », on peut penser que cela renvoie à un usage de son temps, c'est-àdire de la fin du IV siècle av. J.-C. [8]. De fait, on utilise habituellement Athénée pour reconstituer des bribes d'œuvres perdues, mais cette perspective n'est pas ici la nôtre. On s'intéressera plutôt à l'époque d'Athénée, donc aux propos des deipnosophistes eux-mêmes et à la manière dont ils introduisent les citations, la paternité des paraphrases étant plus incertaine : I'auteur et ses personnages usent-ils d'appellations globales permettant d'évoquer en bloc l'ensemble des «Orientaux »?

La réponse, disons-le d'emblée, est négative, s'agissant d'un équivalent littéral du terme «Orientaux ». Sont, en revanche, présents des vocables et expressions se référant à la population d'Asie tels que 'Aoıavoí, « les Asiatiques », oi 'A ıı Yuvaĩkes, « les femmes d'Asie », ou oi tìv 'A oíav

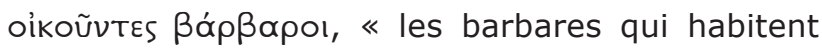
I'Asie » [9]. Pourtant, la moisson reste très maigre (on trouve près de sept occurrences au total dans les quinze livres d'Athénée) et surtout ces expressions ont des chances d'être non pas celles d'Athénée, mais celles des auteurs bien antérieurs qu'il cite. C'est le cas, on l'a dit, de ce passage de Charès de Mytilène où il est question des bijoux faits avec des perles et «que les Perses, les Mèdes et tous les Asiatiques apprécient beaucoup

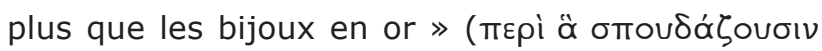

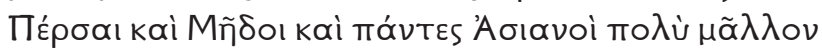

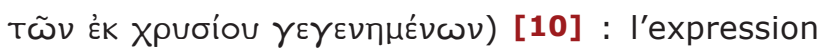
qui introduit ce passage suggère qu'il s'agit d'une citation verbatim [11]. De même, d'après un fragment d'Agatharchide, Lysandre se serait référé aux agissements d'Agésilas vis-à-vis des « natifs

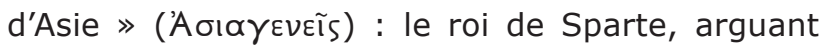

[5] Du moins est-ce la première impression, qui est toutefois superficielle, car, comme l'a noté ZECCHINI 1989, p. 188, Séleucides et Parthes contemporains sont rarement évoqués. La Perse d'Athénée est la Perse achéménide, « un paradigme hors du temps ».

[6] Sur la distinction entre citation et paraphrase sur la base de critères formels, cf. ZEPERNICK 1921 et LENFANT 2007b (les réserves de Peling 2000, tout en n'étant pas sans fondement dans certains cas, reposent sur des exemples qui paraissent exceptionnels une fois replacés dans I'ensemble des citations d'Hérodote. Voir, par ex., LENFANT 2007b, p. 55).

[7] Lenfant 2007b, p. 61 : Athénée prétend qu'Hérodote évoque la truphê de tel personnage, alors que ce dernier n'a pas recours à cette notion précise. Voir aussi Maisonneuve 2007b sur les citations de Xénophon.

[8] III, 93c-d = FGrHist $125 \mathrm{~F} 3$ (voir infra). Sur Charès, voir la récente édition commentée de CAGNAZZI 2015. Sur les fragments de Charès, cf. PAYEN 2007. Sur les références aux Perses chez Charès, voir FisCHer 2011.

[9] Respectivement III, 93d ; XII, 550e ; XIII, 575a-f. Voir aussi ai Ėv Tñ̃ Aớa yuvaĩkes, " les femmes d'Asie 》 (XIII, 609a), oi mepì tìv Aoíav ßápßapol, « les barbares

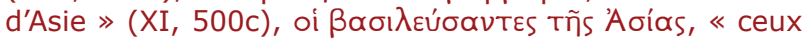
qui ont régné sur l'Asie » (XII, 528e).

[10] III, 93c-d = FGrHist $125 \mathrm{~F} 3$.

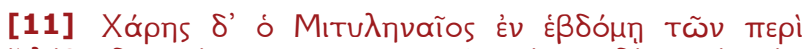

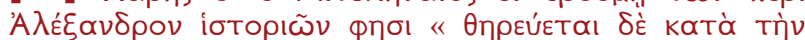

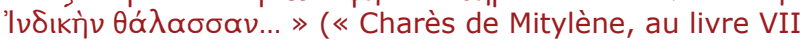
de ses Histoires d'Alexandre, dit: "on pêche dans la mer indienne..." ») : le verbe $\varphi \eta \sigma$, « il dit », est immédiatement suivi d'un fragment de texte qui a gardé jusqu'à la copule $(\delta \varepsilon ́)$ qui le reliait à ce qui le précédait dans I'original. On trouve des exemples analogues parmi les citations d'Hérodote, pour lequel on dispose pour contrôle du texte cité (cf. LeNFANT 2007b, p. 47 et 50-52). 
que ces derniers avaient certes des vêtements de luxe, mais pour cacher des corps sans force, aurait ordonné de mettre séparément en vente les prisonniers et leurs vêtements afin d'afficher à la fois l'intérêt matériel du combat et sa facilité. Dans ce

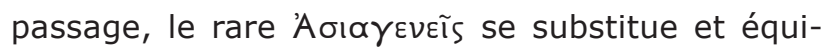
vaut au banal $\beta a ́ p \beta \alpha \rho o$ qui est employé juste avant [12]. Le fragment étant introduit par Ép円 ஸ́s (550d), il constitue selon toute vraisemblance une citation à proprement parler [13]. Il s'agirait alors d'un terme employé par Agatharchide au $\mathrm{II}^{\mathrm{e}}$ siècle av. J.-C. Plus incertain est le cas de ce fragment de Ctésias qui commence par préciser que « tous ceux qui ont régné sur l'Asie ont recherché les plaisirs » [14] : il s'agit certes d'une paraphrase n'excluant pas la reformulation, mais, comme on retrouve cette même référence au règne sur I'Asie chez Diodore (II, 22, 2) quand il s'inspire lui aussi du récit de Ctésias sur les Assyriens [15], il n'est pas exclu que I'expression remonte à I'historien de Cnide (Iv ${ }^{\mathrm{e}}$ siècle av. J.-C.) : ce dernier aurait alors parlé non pas de rois « orientaux » ou de «barbares », mais se serait référé au territoire sur lequel ils régnaient, I'Asie [16]. Quant à I'expression oi mepì tìv Aớav ßápßapol, « les barbares d'Asie », elle apparaît dans ce qui est une citation littérale [17] d'Éphore, autre historien du IV siècle av. J.-C., pour désigner les Perses et leur réputation d'user de ruse et de tromperie à l'époque de I'expédition de Derkylidas en Asie Mineure [18]. On le voit : cette référence aux Asiatiques est peu fréquente et probablement imputable aux sources d'Athénée. En d'autres termes, ce n'est pas à ses yeux une catégorie structurante.

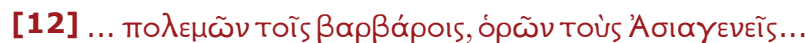
(XII, 550e). Athénée, XII, 550de = FGrHist 86 F 11 .

[13] LenFANT 2007b, p. 50-52.

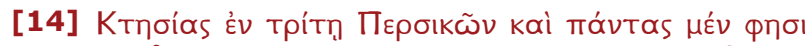

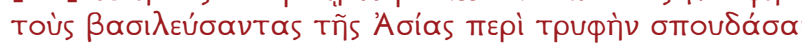
( « Ctésias, au livre trois de ses Persica, affirme que tous ceux qui ont régné sur l'Asie ont recherché les plaisirs (truphê) »). Athénée, XII, 528ef = FGrHist $688 \mathrm{~F} 1 \mathrm{n}$ (LENFANT 2004, p. 69-70).

[15] F 1b § 22, 2 (LENFANT 2004 p. 53).

[16] Comme l'ont rappelé, au sein de ce dossier, Luca Macale et Francesco Mari dans leur article sur le lexique de I'Oriental dans la poésie lyrique et chez Eschyle, «Asie » désignait à l'époque classique le territoire de I'empire perse. De plus, Ctésias représente (à tort) I'empire assyrien comme un strict prédécesseur de l'empire perse sur le plan territorial ( $F$ 1b $\S 2$, 3 et LeNFANT 2004, p. LII-LIII).

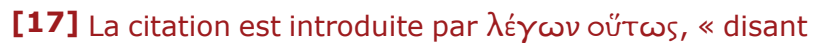
ainsi (= textuellement) », marque d'une citation verbatim. Cf. Lenfant 2007b, p. 51-52.
On s'attend dès lors à voir le terme de « barbares » prédominer en tant que désignation globale des «Orientaux ». Or, s'il est assurément employé, et plus fréquemment que les expressions qui précèdent, il apparaît que ce terme banal est relativement négligé en tant que désignation de l'ensemble des non-Grecs de I'Est méditerranéen. Ses emplois appellent plusieurs types de remarques.

1. Même si de telles statistiques ne sont pas en soi significatives, $\beta \alpha \alpha \rho \beta \alpha \rho o s$ et ses dérivés

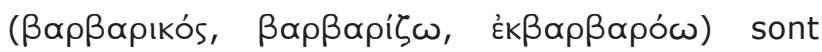
employés moins de cinquante fois dans les quinze livres des Deipnosophistes, un nombre que I'on peut comparer aux quelque 200 occurrences chez Hérodote et 560 chez Plutarque, par exemple. Cela peut, à première vue, étonner dans un ouvrage qui passe en revue les usages de nombreuses communautés grecques et non grecques.

Parmi ces occurrences, certaines figurent dans la bouche des deipnosophistes (13 cas sûrs [19]), mais la plupart apparaissent au sein des paraphrases et citations - ce qui donne à penser qu'elles remontent le plus souvent aux auteurs cités.

2. Plus étonnant : la dichotomie explicite Grecs/ barbares est très rare, et elle ne sert jamais à affirmer une opposition entre les deux catégories [20]. Au contraire, il s'agit presque toujours d'établir une analogie. Cela présuppose certes une distinction préalable, mais l'accent n'en est pas moins mis sur l'équivalence ou la ressemblance, voire l'identité de comportement entre les uns et les autres. Parfois encore, il s'agit de désigner une totalité (les Grecs et les barbares) [21] pour signifier le caractère

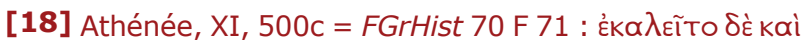

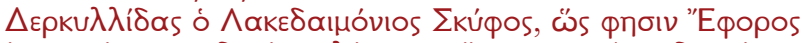

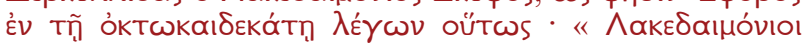

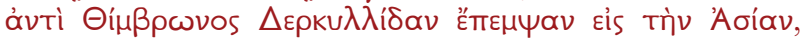

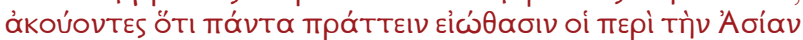

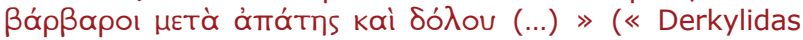
de Lacédémone était appelé Skyphos, comme I'affirme Éphore dans son livre XVIII quand il dit en ces termes : "Les Lacédémoniens envoyèrent Derkylidas en Asie pour remplacer Thibron, parce qu'ils avaient entendu dire que les barbares d'Asie avaient coutume d'agir en tous domaines par tromperie et par ruse" »).

[19] C'est une estimation minimale, qui ne peut être affinée en raison de deux types de difficultés : (1) les deux premiers livres nous sont en fait uniquement connus par un abrégé (épitomé), dans lequel on ne sait pas toujours qui est censé parler ; (2) les méthodes d'Athénée rendent difficile la délimitation des paraphrases.

[20] Par exemple, IV, 167b (dans une citation) ; IV, 184b, XIII, 603a (dans la bouche des deipnosophistes). Cf. $X, 457 f$.

[21] Par exemple, IV, 184b ; XIV, 628c ; 631d. 
répandu, voire universel d'une réalité ou d'une pratique, sans qu'il y ait de hiérarchie affichée.

3. Les affirmations négatives concernant ce qui est «barbare » ( $\beta \alpha ́ \rho \beta \alpha \rho o s$ ou $\beta \alpha \rho \beta \alpha \rho ı$ ós) sont non seulement rares, mais encore toujours situées à I'intérieur de citations et de propos rapportés [22].

4. Sur le plan ethnique et géographique, $\beta \alpha$ ó $\beta \alpha \rho o s$ est souvent un équivalent de «perse » ou sujet du roi de Perse achéménide [23], et c'est non seulement vrai des citations [24], mais aussi parfois des propos des deipnosophistes euxmêmes [25]. C'est qu'alors ils reprennent la tradition d'époque classique. Cependant, il est aussi des cas où $\beta \alpha$ ópapos désigne des non-Grecs d'Occident, Celtes [26], Étrusques [27] ou Gètes [28]. Il n'est donc pas réservé aux « Orientaux ». Notons aussi qu'il n'est pas nécessairement péjoratif et ce n'est pas sans raison que $\beta \alpha$ á $\beta \alpha \rho o s$ ou $\beta \alpha \rho \beta \alpha \rho ı$ sós a été parfois traduit par «local » [29].

5. Une différence importante avec l'époque classique est que ßápßapos n'a pas de contenu politique marqué. L'accent est mis sur les usages et aspects linguistiques et l'on a l'impression que, pour les deipnosophistes eux-mêmes, I'hellénisme est une question de langue, plus que de mœurs [30].

Des interprètes modernes ont cru déceler dans les Deipnosophistes une opposition implicite entre Grecs et barbares, tout particulièrement entre les mœurs des uns et des autres, une opposition qui aurait même joué un rôle dans l'agencement des exemples à l'intérieur de l'ouvrage [31]. Si l'on prend le cas des banquets des divers peuples et cités tels qu'ils sont évoqués dans toute une partie du livre IV (134d155f), on observe que I'organisation ethnico-civique des exemples cités est peu ou prou la suivante. Se succèdent une séquence d'allusions aux repas athéniens (134d-137e), dans laquelle s'insère une allusion aux repas thessaliens (137d), puis des citations relatives aux repas dans la cité de la République de Platon (138a-b), aux repas spartiates (138b143a) - une comparaison avec les repas perses y apparaît au sein d'une citation d'Hérodote (138b) - , aux repas crétois (143a-e), perses (143e-144c, $144 \mathrm{e}-146 \mathrm{c}$ ) - dans cette dernière séquence figure une comparaison avec les repas grecs (144a) - , aux repas d'Alexandre (146c-d) - avec une comparaison avec ceux du roi de Perse-, au repas de Cléopâtre en Cilicie (147e), aux banquets d'Antoine à Athènes et à la tenue dionysiaque de Caligula (148b-d) - cas d'ostentation qui incite à admirer la pauvreté des Grecs. Sont ensuite évoqués les repas des Thébains (148d-e) - en fait, la réception de Mardonios et de 50 Perses par Attaginos -, des Arcadiens (148f149d), de Naucratis (149d-150a), des Égyptiens (150b-d), comparés à ceux des Perses (150b-c), des Galates (150d-f), des Thraces (150f-151e), des Celtes (151f-152f), des Parthes (152f-153a), de rois séleucides (153a-153c), des repas à Rome lors des triomphes $(153 c-d)$, des repas des Étrusques (153d), des Indiens (153d-e), des Germains, des Campaniens (153d), des Romains (153f-154a), des Étrusques (154a), des Celtes (154a-c), les spectacles de gladiateurs chez les Romains et les danses en armes chez les rois hellénistiques (154c-155c), les banquets d'Alexandre, des Thraces, et l'absence de banquets trop arrosés à Sparte (155c-156a).

Ce sommaire, qui gomme assurément de nombreuses subtilités de cette longue séquence, est loin de suggérer que le fil rouge de l'ensemble soit I'opposition Grecs-barbares. Certes, I'auteur introduit la séquence sur les repas perses en disant qu'Hérodote a comparé les banquets des Grecs à ceux
[22] XI, 461b-d (où l'opposition est du reste largement nuancée) ; XI, 500c ; éventuellement XII, 512b ; XIV, $632 a-b ; X V, 672 d$. En VIII, 350a, on observe un cas exceptionnel d'emploi négatif, mais dans un sens qui n'est pas ethnique, mais moral (les personnages concernés sont des Grecs, les Béotiens et les Thessaliens).

[23] Plus rarement, la référence est aux Parthes : IV, 153a-b.

[24] VI, 252b, citation de Posidonios ; XI, 500c, citation d'Éphore ; XII, 538e, citation de Charès ; XIII, 575f, citation de Charès etc.

[25] I, 29f-30a (deipnosophiste non nommé dans l'Épitomé ; VI, 253f (deipnosophiste Démocrite moralisant sur les flatteurs).

[26] IV, 152a (dans une citation).

[27] XII, 518a (dans une citation).

[28] En XIV, 627d, la citation de Théopompe évoque les Gètes, mais l'introduction par le deipnosophiste désigne les barbares.
[29] Voir, dans Ateneo 2001, la traduction du mot en IV, $152 \mathrm{a}$ et $153 \mathrm{~b}$ par Leo Citelli, en XI, 784b par Rodolfo CHERUBINA.

[30] Cf. VI, 229a.

[31] Bruit \& Schmitt Pantel 1986, Schmitt Pantel 1992 , p. 421-438, sur l'exemple des banquets au livre IV, NADEAU 2010, s'intéressant au livre XII, se réfère à Bruit \& SChmitT Pantel 1986, p. 204 et 220-221, qui sont cependant plus subtiles qu'il ne le laisse entendre (p. 366 : «Comme I'ont montré Louise Bruit Zaidman et Pauline Schmitt Pantel dans leur analyse du livre IV, les habitudes alimentaires attribuées aux populations vivant en marge du monde hellène sont souvent décrites à partir de préjugés ethnographiques stéréotypés. En cela, Athénée et ses auteurs contemporains (sic) demeurent tributaires de ces topoi littéraires; ainsi, les récits de pratiques étrangères les aident à penser les pratiques alimentaires gréco-romaines. »). La distinction entre les deipnosophistes et les auteurs qu'ils citent n'est pas prise en compte. 
des Perses (143f) et l'on peut voir qu'ici et là les repas de ces derniers sont cités comme étant I'opposé de ceux des Spartiates ou plus généralement des repas « pauvres » des Grecs. Pourtant, ils sont tout aussi bien comparés à ceux d'Alexandre ou des Égyptiens. Ces comparaisons ne correspondent donc pas toujours à une antithèse Grecsbarbares et font en outre presque toujours partie des citations : ce sont celles des sources d'Athénée. Qui plus est, les extraits passent de tel groupe grec à tel groupe non grec sans qu'il y ait solution de continuité. Du reste, les enchaînements d'exemples sont parfois imputables non pas tant à l'évolution de la thématique qu'au fait qu'Athénée emprunte plusieurs citations successives à un même auteur, Posidonios, Théopompe ou Phylarque, par exemple. Par exemple, la séquence 151e-153d, qui voit se succéder des citations sur les Celtes, sur les Parthes, sur les Séleucides, sur Rome et sur les Étrusques est en fait entièrement constituée de citations tirées de Posidonios : c'est le phénomène des clusters de citations bien mis en lumière par Christopher Pelling, qui précise : «a particular author can be used to provide the skeletal framework for a whole section » [32]. Au total, il faut se livrer à une rude gymnastique pour considérer que la distinction Grecs-barbares (ou la distinction Est-Ouest) structure le propos d'Athénée, y compris de manière implicite.

De fait, ce n'est pas un hasard si, contrairement à d'autres collectionneurs d'anecdotes comme Polyen ou Valère Maxime, Athénée n'a pas fait le choix de regrouper les exempla barbares et de les mettre à part comme distincts et homogènes. Il n'y a pas trace dans son œuvre d'une opposition générale entre Grecs et barbares, encore moins entre les Grecs et «les Autres ». Le constat se confirme si I'on prend en compte les thématiques : la truphê n'est absolument pas présentée comme une exclusivité des barbares, même si ces derniers en fournissent les exemples les plus frappants [33]. Certes, les termes de Grecs et de barbares sont présents, mais de manière résiduelle, principalement à l'intérieur des citations. Encore l'héritage est-il sélectif, surtout si on le compare à la littérature d'époque classique, où la dimension politique des mœurs jouait un rôle important dans l'antithèse.
Au vrai, faut-il s'en étonner ? Athénée n'a pas de penchant particulier pour la démocratie et n'a rien contre la monarchie. Comme I'a montré Giuseppe Zecchini, il a peu d'estime pour la Grèce classique et singulièrement pour Athènes, alors qu'il est manifestement séduit par la monarchie lagide telle qu'elle était au III $^{\mathrm{e}}$ siècle av. J.-C. [34] - une monarchie dont il ne faut pas oublier qu'elle avait su reprendre des pratiques jugées antérieurement caractéristiques des Perses, au point même de revendiquer parfois la truphê jusque dans le surnom officiel du roi [35].

Du reste, si les deipnosophistes ne s'identifient pas à une norme grecque en matière d'usages, c'est d'abord qu'il n'y en a pas vraiment dans les domaines qui l'intéressent. L'ouvrage donne à voir des mœurs qui sont diverses parmi les Grecs comme parmi les barbares, et varient tantôt d'une communauté à I'autre, tantôt à l'échelle individuelle. Quel que soit leur caractère fictif [36], la diversité d'origine des deipnosophistes est en soi tout un symbole : Élée, la Thessalie, Nicée, Pergame, Éphèse, Nicomédie, Tyr, Alexandrie, Naucratis, Ptolémaïs, la Mauritanie et Rome. L'origine rejaillit parfois explicitement sur le point de vue de certains d'entre eux : Athénée, de Naucratis, non loin d'Alexandrie, évoque Ptolémée

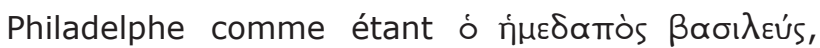
«le roi de chez nous » (I, 3b), mais, quand c'est

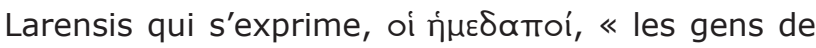
chez nous », sont de toute évidence les Romains (VI, 273a). De fait, I'arrière-plan romain ne doit pas être négligé en tant que référence implicite : d'après l'abréviateur, Larensis, « en invitant à ses festins, fait à tous de Rome une patrie » (I, 3c :

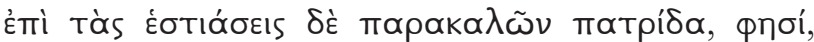

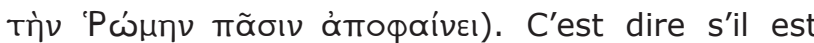
délicat de juger les usages barbares comme étant les usages « des autres», alors que bien souvent les usages des Grecs ne sont pas moins ceux des « autres ». Du reste, ce n'est pas un hasard si les pratiques des lieux d'origine des deipnosophistes sont parfois précisées : on voit Cynulcus interpeller Plutarque en tant qu'Alexandrin, originaire d'une ville où abondent les plats à base de lentilles (IV, 158d), tandis que Démocrite plaisante Ulpien parce qu'en raison de ses origines syriennes il aurait privé ses compagnons de poisson (VIII, 346c). Car
[32] Pelling 2000, p. 174-175.

[33] LeNFANT 2007a, p. 53.

[34] ZeCCHini 1989, p. 37 et 154 , et 2007 , p. 26.
[35] Sur le surnom de Tryphôn pris par plusieurs Ptolémées, cf. Heinen 1983.

[36] Sur les personnages de deipnosophistes, cf. ROMERI 2002, p. 278-282. 
Ulpien, le puriste de la langue attique, n'est autre qu'un Syrien.

Pour conclure, alors qu'il vit à l'époque de la Seconde Sophistique, dont la rhétorique a volontiers exacerbé I'opposition culturelle entre Grecs et barbares, tant sur le plan linguistique et littéraire que moral, Athénée, qui n'est pas sans affinités avec ce courant [37], contraste fortement néanmoins avec un Aristide ou un Plutarque, chez qui la figure du barbare est un contrepoint plus stéréotypé que jamais sur le plan moral [38]. À l'inverse de cet auteur, chez qui Thomas Schmidt relève « I'absence d'une véritable différenciation entre les peuples barbares »[39], les Deipnosophistes, malgré l'inévitable présence de clichés hérités sur les barbares, ne distinguent pas un bloc de barbares ou d'Orientaux qui s'opposeraient aux Grecs (et aux Romains), y compris quand I'auteur consacre tout un livre à un thème d'allure aussi « orientaliste » que la truphê. La distinction d'un ensemble humain, oriental ou non, qui s'opposerait aux locuteurs et à leurs modèles ne trouve de manifestation ni lexicale ni structurelle ni thématique. L'hellénisme célébré par l'auteur est celui de la langue et de la littérature qui alimentent les convives en citations dont ils se régalent à satiété, mais la sélection opérée dans cette littérature correspond plus à la curiosité universelle d'un anthropologue qu'à la conviction d'une dichotomie générale de I'humanité [40].

[37] Mainguy 2011, p. 120. Le même auteur commente l'atticisme dont témoignent les Deipnosophistes tout en raillant ses excès (p. 122-128).

[38] Sur Aelius Aristide, cf. la contribution de Jean-Luc Vix dans ce dossier. Sur Plutarque, cf. SCHMIDT 1999 et 2002.

[39] SCHMIDT 1999, p. 332.

[40] Sur Athénée comme anthropologue, cf. JACOB 2001.

\section{BIBLIOGRAPHIE}

Ateneo. I deipnosofisti. I dotti a banchetto, 2001, prima traduzione italiana commentata su progetto di Luciano Canfora, 4 vol., Roma.

Bruit, Louise \& Schmitt Pantel, Pauline, 1986, «Citer, classer, penser : à propos des repas des Grecs et des repas des autres dans le livre IV des Deipnosophistes d'Athénée », AION 8, p. 203-211.

Cagnazzi, Silvana, 2015, Carete di Mitilene. Testimonianze e frammenti, Tivoli (Roma).

Fischer, Fabrice, 2011, « Charès de Mytilène », dans Dominique Lenfant (éd.), Les Perses vus par les Grecs. Lire les sources classiques sur l'empire achéménide, Paris, p. 88-91.

Gambato, Maria, 2000, "The Female-Kings: Some Aspects of the Representation of Eastern Kings in Athenaeus' Deipnosophistae », dans David Braund \& John Wilkins (éd.), Athenaeus and his World, Exeter, p. 227-230.

Heinen, Heinz, 1983, « Die Tryphè des Ptolemaios VIII. Euergetes II. Beobachtungen zum ptolemäischen Herrscherideal und zu einer römischen Gesandschaft in Ägypten (140/39 v. Chr.) », dans Heinz Heinen (éd.), Althistorische Studien H. Bengtson, Historia Einzelschriften, 40, p. 116-128.

JАсов, Christian, 2001, «Ateneo o il dedalo delle parole », dans Ateneo. I deipnosofisti. I dotti a banchetto, prima traduzione italiana commentata su progetto di L. Canfora, 4 vol., Roma, p. XI-CXVI.

JACOB, Christian, 2013, The Web of Athenaeus, Washington.

KaIBeL, Georg, 1887-1890, Athenaei Naucratitae Dipnosophistarum libri XV, 3 vol., Teubner, Leipzig.

Lenfant, Dominique, 2004, Ctésias de Cnide. La Perse. L'Inde. Autres fragments, texte édité, traduit et commenté, CUF, Paris.

Lenfant, Dominique, 2007a, « On Persian tryphè in Athenaeus », dans Christopher Tuplin (éd.), Persian Responses. Political and Cultural Interaction with(in) the Achaemenid Empire, Swansea, p. 51-65.

Lenfant, Dominique, 2007b, « Les «fragments» d'Hérodote dans les Deipnosophistes », dans Dominique Lenfant (éd.), Athénée et les fragments d'historiens, Paris, p. 43-72.

Lenfant, Dominique, 2011, « Athénée de Naucratis», dans Dominique Lenfant (éd.), Les Perses vus par les Grecs. Lire les sources classiques sur l'empire achéménide, Paris, p. 65-83.

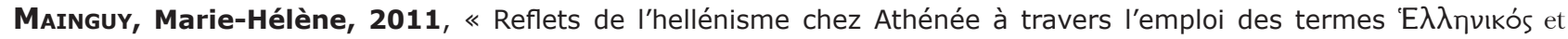
ßápßapos », dans Thomas Schmidt \& Pascale Fleury (éd.), Perceptions of the Second Sophistic and its Times - Regards sur la Seconde Sophistique et son époque, Toronto, p. 120-132.

Maisonneuve, Christine, 2007, «Les "fragments" de Xénophon dans les Deipnosophistes », dans Dominique Lenfant (éd.), Athénée et les fragments d'historiens, Paris, p. 73-106. 
NAdeAU, Robin, 2010, Les manières de table dans le monde gréco-romain, Rennes.

Olson, Douglas, 2006-2012, Athenaeus. The Learned Banqueters, 8 vol., Loeb, Cambridge (Mass.) - London.

Payen, Pascal, 2007, «Les fragments de Charès de Mytilène chez Athénée », dans Dominique Lenfant (éd.), Athénée et les fragments d'historiens, Paris, p. 191-214.

Pelling, Christopher, 2000, « Fun with Fragments. Athenaeus and the Historians », dans David Braund \& John Wilkins (éd.), Athenaeus and his World, Exeter, p. 171-190.

Romeri, Luciana, 2002, Philosophes entre mots et mets. Plutarque, Lucien et Athénée autour de la table de Platon, Grenoble.

Schmidt, Thomas, 1999, Plutarque et les Barbares. La rhétorique d'une image, Louvain - Namur.

Schmidt, Thomas, 2002, «Plutarch's Timeless Barbarians and the Age of Trajan », dans Philip A. Stadter \& Luc Van der Stockt (éd.), Sage and Emperor. Plutarch, Greek Intellectuals, and Roman Power in the Time of Trajan (98-117 A.D.), Leuven, p. 57-71.

Schmitt Pantel, Pauline, 1992, La cité au banquet. Histoire des repas publics dans les cités grecques, Rome.

Zecchini, Giuseppe, 1989, La cultura storica di Ateneo, Milano.

Zесchini, Giuseppe, 2007, « Athénée et les historiens : un rapport indirect », dans Dominique Lenfant (éd.), Athénée et les fragments d'historiens, Paris, p. 19-28.

ZePeRnICK, Kurt, 1921, «Die Exzerpte des Athenaeus in den Dipnosophisten und ihre Glaubwürdigkeit», Philologus 77, p. 311-363. 